\title{
Magnetic phase diagram of the high-temperature spiral magnet $\mathrm{YBaCuFeO}_{5}$
}

\author{
J. Lyu ${ }^{1}$, T. Shang ${ }^{1,2}$, M. Morin ${ }^{1,3}$, M. T. Fernández-Díaz ${ }^{4}$ M. Medarde ${ }^{1}$ \\ ${ }^{1}$ Laboratory for Multiscale Materials Experiments, Paul Scherrer Institut, 5232 Villigen PSI, Switzerland, ${ }^{2}$ Key Laboratory of Polar \\ Materials and Devices (MOE), School of Physics and Electronic Science, East China Normal University, Shanghai 200241, China, \\ ${ }^{3}$ Excelsus Structural Solutions (Swiss) AG, PARK innovAARE, 5234 Villigen, Switzerland, ${ }^{4}$ Institut Laue Langevin, 71 avenue des
}

Martyrs, CS 20156, 38042 Grenoble CEDEX 9, France

jike.lyu@psi.ch

Frustrated magnets with spiral magnetic phases are currently being intensively studied owing to their ability for inducing ferroelectricity. This could potentially be exploited in spintronics and low power memories devices.[1-2] However, the low magnetic order temperatures (typically $<100 \mathrm{~K}$ ) in most of frustrated magnets greatly restrict their fields of application. One of the most notable exceptions are $\mathrm{Cu} / \mathrm{Fe}$-based layered perovskites, featuring magnetic spiral phases whose ordering temperatures can be continuously tuned far beyond RT. [3-5]. However, the influence of magnetic field on the magnetic structures especially spiral phases, imperative for further cross-control of the magnetic and ferroelectric orders, is barely known.

Here, we report a comprehensive description of the evolution of magnetic order in the layered perovskite $\mathrm{YBaCuFeO}_{5}$ under the application of magnetic fields up to $9.0 \mathrm{~T}$ and at temperatures between $1.5 \mathrm{~K}$ and $300 \mathrm{~K}$. Using bulk magnetization measurements and neutron powder diffraction we reveal the existence of a new incommensurate magnetic phase with a weak ferromagnetic component stable at low magnetic fields. Moreover, we observe a field-induced spin reorientation in the collinear phase. The resulting $H-T$ phase diagram of $\mathrm{YBaCuFeO}_{5}$ will be discussed, with emphasis in the magnetic phases with the largest potential to display strong magnetoelectric effects. [6]
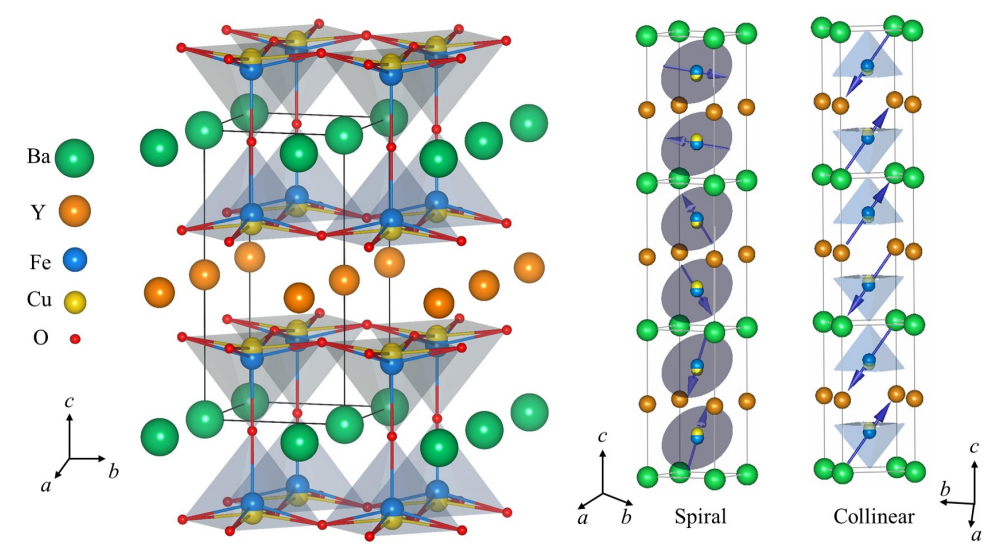

Figure 1. Left: Crystal structure of $\mathrm{YBaCuFeO}_{5}$ showing the $\mathrm{Cu} / \mathrm{Fe}$ disorder in the bipyramidal sites. Magnetic structure of the incommensurate spiral phase (Middle) and the commensurate collinear phase (Right).

[1] Eerenstein, W., Mathur, N.D. \& Scott, J.F. (2006). Nature. 442, 759.

[2] Kimura, T., Goto, T., Shintani, H., Ishizaka, K., Arima, T.H. \& Tokura, Y. (2003). Nature 426, 55.

[3] Morin, M., Scaramucci, A., Bartkowiak, M., Pomjakushina, E., Deng, G., Sheptyakov, D., Keller, L., Rodriguez-Carvajal, J., Spaldin, N.A., Kenzelmann, M., Conder, K. \& Medarde, M. (2015). Phys. Rev. B 91, 064408.

[4] Morin, M., Canévet, E., Raynaud, A., Bartkowiak, M., Sheptyakov, D., Ban, V., Kenzelmann, M., Pomjakushina, E., Conder, K. \& Medarde, M. (2016). Nat. Commun. 7, 1.

[5] Shang, T., Canévet, E., Morin, M., Sheptyakov, D., Fernández-Díaz, M. T., Pomjakushina, E. \& Medarde, M. (2006). Sci. Adv. 4, eaau6386.

[6] Lyu, J. et al. in preparation.

Keywords: YBaCuFeO5; High-temperature Magnetic Spiral; Magnetic Phase Diagram; Multiferroic

Acta Cryst. (2021), A77, C407 